\title{
Quantum-confined superfluid: From nature to artificial
}

\author{
Liping Wen ${ }^{1,2}$, Xiqi Zhang ${ }^{1}$, Ye $\operatorname{Tian}^{2,3}$ and Lei Jiang ${ }^{1,2,4^{*}}$
}

\begin{abstract}
Biological ion channels show that ultrafast ions and molecules transmission are in a quantum way of single molecular or ionic chain with a certain number of molecules or ions, and we define it as "quantum-confined superfluid" (QSF). This ordered ultrafast flow in the confined channel can be considered as "quantum tunneling fluid effect" with a "tunneling distance", which is corresponding to the period of QSF. Recent research demonstrated that artificial biomimetic nanochannels also showed the phenomenon of QSF, such as ion and water channels. The introduction of QSF concept in the fields of chemistry and biology may create significant impact. As for chemistry, the QSF effect provides new ideas for accurate synthesis in organic, inorganic, polymer, etc. We believe the implementation of the idea of QSF will promote the development of QSF biochemistry, biophysics, bioinformatics and biomedical science.
\end{abstract}

Keywords: quantum-confined superfluid, quantum tunneling fluid effect, ion channels, artificial nanochannels

Superfluid is a cross-century topic, which was originally discovered in ${ }^{4} \mathrm{He}$ below $2.17 \mathrm{~K}$, by Kapitsa and Allen $[1,2]$. The viscosity of ${ }^{4} \mathrm{He}$ superfluid was measured by Kapitsa, as the liquid flowed through the gap between two glass disks with distance of $\sim 500 \mathrm{~nm}$, which showed the viscosity was at least 1,500 times lower than that of ${ }^{4} \mathrm{He}$ at normal pressure, and $10^{4}$ times lower than that of hydrogen gas [1]. This superfluid with zero viscosity allows liquid to flow without loss of kinetic energy. Allen reported ${ }^{4} \mathrm{He}$ superfluid through capillaries with varying channel diameters, and found the velocity of superfluid increased rapidly with decreasing channel size [3]. In a capillary with an intrinsic diameter less than $100 \mathrm{~nm}$, the fluid velocity was completely independent of pressure and the channel length, but only depending on the temperature.

Interestingly, similar phenomenon also exists in biological system. Biological ion channels embedded in plasma membranes play important roles in a wide spectrum of physiological processes such as mass transfer, energy conversion and signal transmission [4]. Taste perception, for example, functions because calcium homeostasis modulator can open and release adenosine triphosphate (ATP) rapidly when the flavorous molecules, such as sour, sweet, bitter and salty, touch its taste receptor [5]. Electric eel can generate potential differences of over $600 \mathrm{~V}$ with electrically active cells known as electrocytes, while the time of each electrification process is in the millisecond magnitude, owing to the ultrafast ion transport across the $\mathrm{Na}^{+}$and $\mathrm{K}^{+}$channels [6]. On the basis of biological ion channels, signals can be transmitted from the nerves to brain in the process of vision, smell, audition and tactility. Those crucial functions highly depend on their selective protein-based gatekeepers that allow extremely rapid transit $\left(10^{7}\right.$ ions per channel in one second) [7]. This ultrafast mass transfer stems from the special features of the biological ion channels, e.g., small size, unique structure and surface charge distribution, which lead to peculiar properties, inducing ultrafast ion and molecule transmission in the form of single strand.

From the viewpoint of classical thermodynamics, mass transport across nanometer-scale channel with chemical selectivity should be very slow: this limitation is predicted by the Hagen-Poiseuille equation and it is because conventional laminar flow has zero fluid velocity at the pore

\footnotetext{
${ }^{1}$ Key Laboratory of Bio-inspired Materials and Interfacial Science, Technical Institute of Physics and Chemistry, Chinese Academy of Sciences, Beijing 100190, China

${ }^{2}$ University of Chinese Academy of Sciences, Beijing 100049, China

${ }^{3}$ Beijing National Laboratory for Molecular Sciences (BNLMS), Key Laboratory of Green Printing Institute of Chemistry, Chinese Academy of Sciences, Beijing 100190, China

${ }^{4}$ Key Laboratory of Bio-inspired Smart Interfacial Science and Technology of Ministry of Education, School of Chemistry, Beihang University, Beijing 100191, China

*Corresponding author (email: jianglei@iccas.ac.cn)
} 

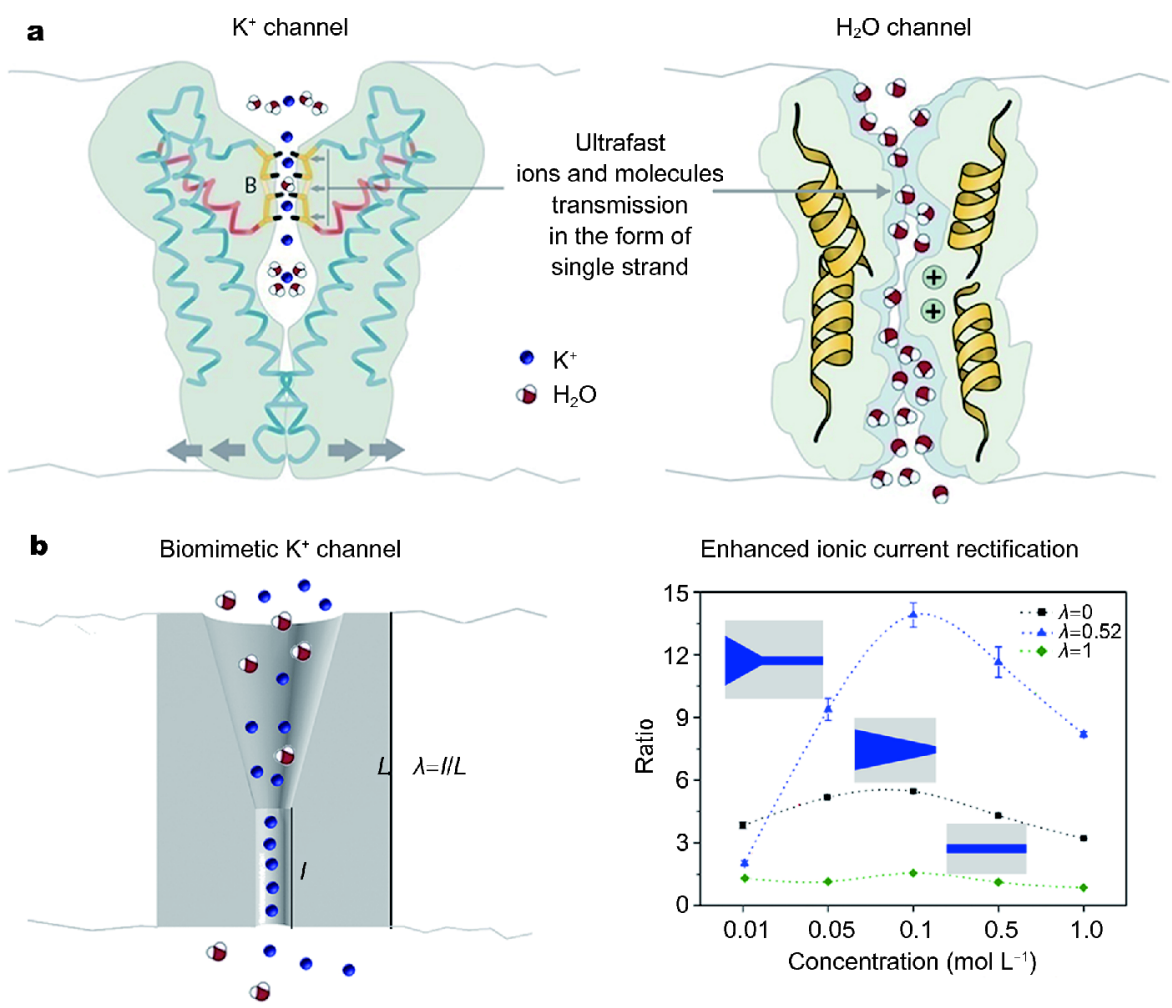

Enhanced ionic current rectification

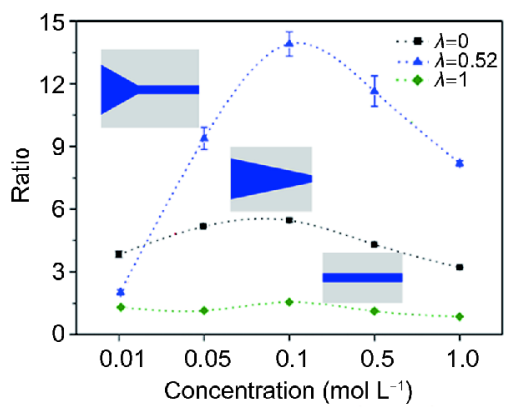

Figure 1 Ultrafast ions and water transmission exist both in biological and artificial ion channels. (a) A biological $\mathrm{K}^{+}$channel contains two $\mathrm{K}^{+}$ions about 7.5 angstroms apart with a single water molecule in between, and a biological water channel contains an ordered water molecular strand, indicating the ultrafast transit is in a quantum way, i.e., "quantum-confined superfluid" (QSF). Reproduced with permission from Ref. [11], Copyright 2004, Wiley-VCH. (b) Biomimetic artificial nanochannels have tremendous rectification with an increased critical region of the cylindrical segment, whose asymmetric rectifying behavior was associated with the gradual structure and caused by the quantized flow, indicating the possibility of artificial QSF systems. Reproduced with permission from Ref. [14], Copyright 2016, Wiley-VCH.

walls [8]. In the living system, however, the fast transit of ions and molecules is precisely the state of ultrafast fluid caused by a quantized flow. For example, the NaK nonselective channel enables only one fully hydrated $\mathrm{Na}^{+}$ion to reside in the selectivity filter [9]; the potassium filter from Streptomyces lividans contains two $\mathrm{K}^{+}$ions about 7.5 angstroms apart, with a single water molecule in between (Fig. 1a) $[10,11]$; and in calmodulin, each calcium channel also simultaneously binds two $\mathrm{Ca}^{2+}$ ions [12]. These phenomena indicate that the ultrafast transit is in a quantum way of single molecular or ionic chain with a certain number of molecules or ions, i.e., "quantumconfined superfluid" (QSF). By imitating biological ion channels, our group has started the work of synthesizing smart gating nanochannels by modifying smart molecules onto the inner surface of micro/nano confined channels since 2008 [13]. And tremendous rectification with an increased critical region of the cylindrical segment was achieved in the nanochannels, whose asymmetric rectifying behavior was associated with the gradual structure and caused by the quantized flow (Fig. 1b) [14], indicating the possibility of artificial QSF systems.

The QSF effect exhibits not only in ion transport, but also in the water molecule transport. During the past decade, researchers found that the ultrafast water flow through aligned carbon nanotube (CNT) membrane is four to five orders of magnitude faster than that predicted from conventional fluid-flow theory $[8,15,16]$. Both experimental results and simulations have demonstrated that the confined water flux could decrease one order of magnitude in the hydrophilic nanochannels, whereas it could increase up to seven orders of magnitude in the hydrophobic nanochannels compared with that of bulk water (Fig. 2a, b) [17]. In the case of molecular dynamics (MD) simulation, Hummer and coworkers reported spontaneous and continuous filling of a hydrophobic CNT with a one-dimensionally ordered chain of water molecules (about five water molecules) and observed pulse-like transmission of water through the nanotube (Fig. 2c) [18]. It should be noted here, they ignore the fact 

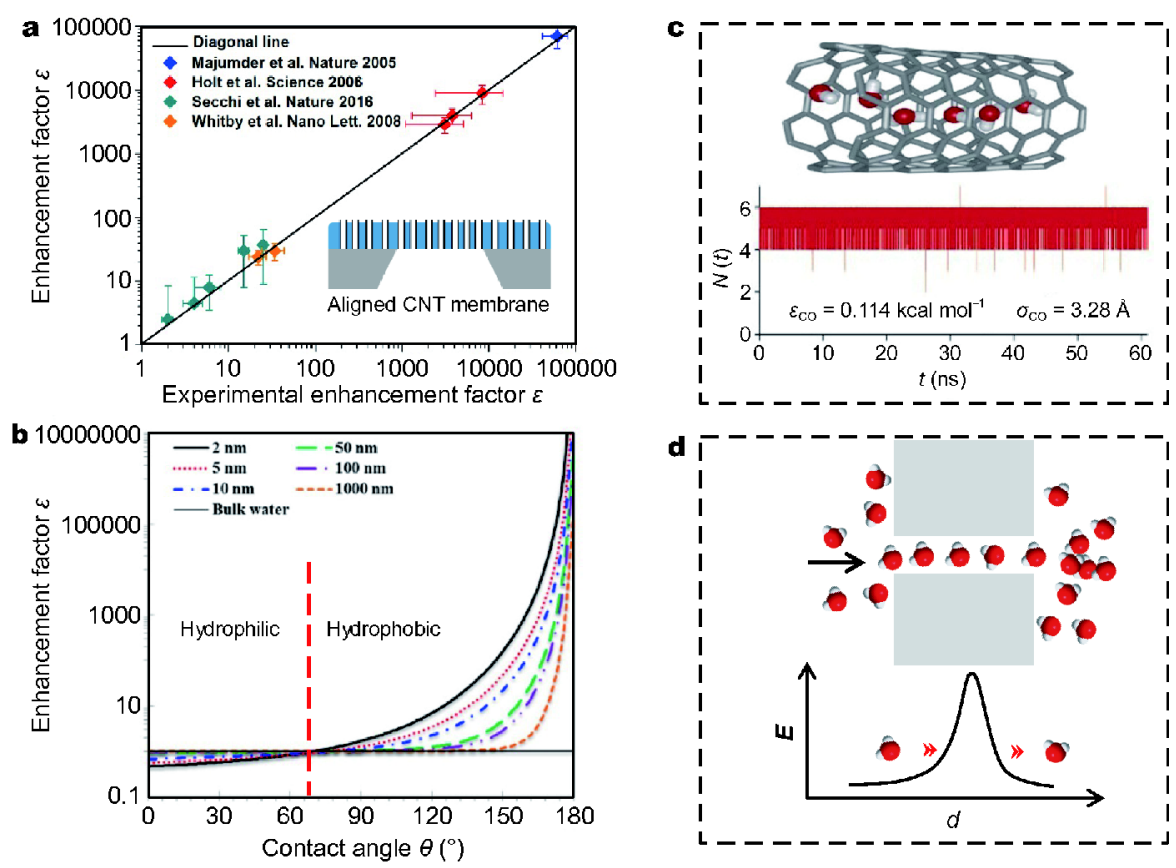

Figure 2 Ultrafast water transmission in artificial 1D nanochannels and the proposed "quantum tunneling fluid effect". (a) Comparison of water flow enhancement factors calculated by experimental data in the literature. Inset represents aligned CNT membrane. (b) The dependence of enhancement factor of various nanochannel diameters on contact angle. Reproduced with permission from Ref. [17], Copyright 2017, National Academy of Sciences, USA. (c) Structure of the hydrogen-bonded water chain inside the carbon nanotube by MD simulation and number of water molecules inside the nanotube as a function of time. Reproduced with permission from Ref. [18] Copyright 2001, Nature Publishing Group. (d) Schematic representation of ultrafast water transport through the nanochannel with an ordered water molecular strand, and the proposed "quantum tunneling fluid effect".

that the inlet of CNT is hydrophilic [15], although no influence on their results. Our simulation shows that water can only penetrate through the nanotube with hydrophilic inlet [19]. From the classical hydrodynamics, the penetration barrier in such a small channel in both biological and artificial systems is high, and the ordered ultrafast flow in the confined channel can be considered as "quantum tunneling fluid effect" (Fig. 2d). Therefore, there should exist a "tunneling distance", which is corresponding to the period of QSF. As for potassium ions and water channels, the lengths of tunneling distance are the size of two $\mathrm{K}^{+}$ions with a single water molecule in between and water molecular strand, respectively.

Based on the above idea, we have designed two-dimensional hydrophilic/hydrophobic alternative nanodomain network surface, and the QSF effect of various liquids could be obtained, exhibiting super-amphiphilicity and superspreading in macroscopic scale [20]. The as-prepared silicon wafer shows a superamphiphilic surface, i.e., both water and other organic solvents are superspreading on the surface (Fig. 3a). Another example is two-dimensional graphene oxide (GO) nanochannels. Nair et al. [21] reported GO (with hydrophilic end-

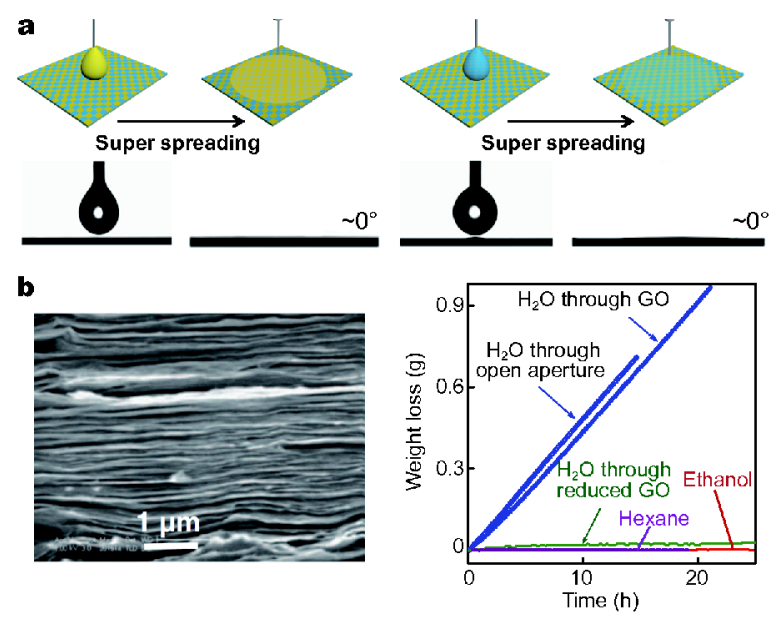

Figure 3 Superspreading of liquids in 2D surface and ultrafast water transport in 2D interfacial systems. (a) Schematic description of the mechanism of super-amphiphilic silicon wafer as a proof of concept in QSF. Hydrophilic and hydrophobic nanodomains network co-existing upon the silicon wafer, and both oil (hexane) and water are superspreading on the surface. (b) Electron micrograph (left) of the GO membrane's cross section and graphic (right) demonstrating ultrafast water flow through GO membrane. Reproduced with permission from Ref. [21], Copyright 2012, American Association for the Advancement of Science. 


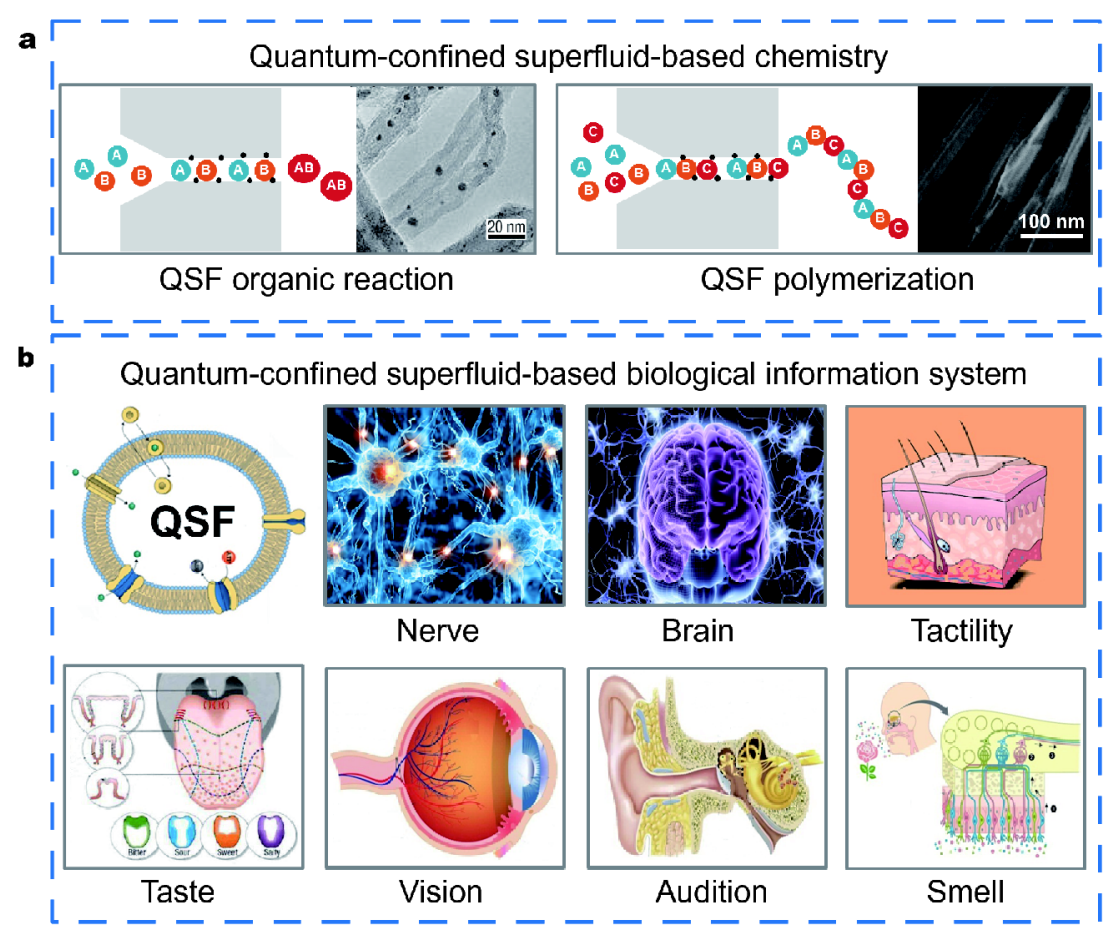

Figure 4 The introduction of QSF concept in chemistry and biology may create QSF chemistry and biology. (a) QSF organic reaction and QSF polymerization: Fischer-Tropsch synthesis confined in CNTs can increase synthetic yield by an order of magnitude; and crystalline nanofibers of linear polyethylene with an ultrahigh molecular weight and higher density can be synthesized. Reproduced with permission from Ref. [31], Copyright 2007, Nature Publishing Group and Ref. [32], Copyright 1999, American Association for the Advancement of Science. (b) The QSF concept is of great significance for deep understanding ultrafast signal transit of nerves and brains, and will provide new ideas for understanding the physiological processes, including tactility, taste, vision, audition and smell, and promote the development of QSF biochemistry, biophysics, bioinformatics and biomedical science.

group) membranes allowed ultrafast water permeation (Fig. 3b), but no permeation in the reduced GO. These are good examples for demonstration of the QSF on 2D surface and interfacial systems.

Besides fundamental researches, technology developments have also been carried out on the bio-inspired energy conversion systems. Our group has developed an energy conversion system based on a QSF single channel, which could generate considerable bioelectricity from the salt content in fluids, and the maximum power output $\left(\left(P_{\mathrm{L}}\right)_{\max }\right)$ from individual nanopore reached $26 \mathrm{pW}$ [22]. Besides, boron nitride nanotube and single-layer molybdenum disulfide $\left(\mathrm{MoS}_{2}\right)$ nanopores have also been used to develop energy conversion devices, which achieved very high power density due to the QSF effect $[23,24]$. Furthermore, using the QSF effect, a series of large-scale nanochannel membranes have been fabricated and used in salinity gradient driven energy conversion systems, whose power density has almost meet the commercial standard [25-27]. As for next remaining challenge, imitating ATP energy conversion and storage process will enable us to synthesize similar functional molecules and smart membrane to realize biomimetic chemical energy storage and conversion (Personal communication with Prof. Markus Antonietti, Max Planck Institute of Colloids and Interfaces, Germany, March, 2018).

Furthermore, the introduction of QSF concept in the field of chemistry may create QSF chemistry (organic, inorganic, polymer, analysis, etc.). By imitating enzyme synthesis, this quantum superfluid, which arrange the reactant molecules in a certain order, will greatly reduce the reaction barrier and achieve highly efficient and selective chemical synthesis, such as QSF catalysis. QSF catalysis has been realized in the biosystem, i.e., the enzyme synthesis [28], DNA synthesis [29], and it was also extended to artificial synthesis of polybutadiene [30]. In this regard, some previous studies approached the example of QSF-like chemical reactions, including organic synthesis: Bao and coworkers [31] reported FischerTropsch synthesis confined in CNTs could increase synthetic yield by an order of magnitude; and polymer synthesis: Aida and coworkers [32] reported crystalline nanofibers of linear polyethylene with an ultrahigh mo- 
lecular weight and higher density (Fig. 4a). In the field of biology, almost all the living processes are completed by ion and molecule channels. The introduction of QSF will provide new ideas for understanding the scientific nature of these physiological processes, and development of QSF biochemistry, biophysics, bioinformatics and biomedical science. Especially, it is of great significance for deep understanding ultrafast signal transit between the nerves and brain (Fig. 4b), which might be based on QSF ion waves. In conclusion, the application prospect based on the concept of "QSF" should be very broad in the field of chemistry and biology, and will further promote the development of new materials, energy conversion method, information system and medical science.

\section{Received 21 April 2018; accepted 24 April 2018; \\ published online 2 May 2018}

1 Kapitza P. Viscosity of liquid helium below the $\lambda$-point. Nature, 1938, 141: 74

2 Allen JF, Misener AD. Flow of liquid helium II. Nature, 1938, 141: 75

3 Allen JF, Misener AD. The properties of flow of liquid He II. Proc R Soc A-Math Phys Eng Sci, 1939, 172: 467-491

4 Hille B. Ionic channels of excitable membranes, $3^{\text {rd }}$ Edition. Sunderland: Sinauer Associates, 2001

5 Taruno A, Vingtdeux V, Ohmoto M, et al. CALHM1 ion channel mediates purinergic neurotransmission of sweet, bitter and umami tastes. Nature, 2013, 495: 223-226

6 Xu J, Lavan DA. Designing artificial cells to harness the biological ion concentration gradient. Nat Nanotechnol, 2008, 3: 666-670

7 Sansom MSP, Shrivastava IH, Bright JN, et al. Potassium channels: structures, models, simulations. BioChim Biophysica Acta (BBA)Biomembranes, 2002, 1565: 294-307

8 Majumder M, Chopra N, Andrews R, et al. Enhanced flow in carbon nanotubes. Nature, 2005, 438: 44-44

9 Shi $\mathrm{C}, \mathrm{He} \mathrm{Y}$, Hendriks $\mathrm{K}$, et al. A single $\mathrm{NaK}$ channel conformation is not enough for non-selective ion conduction. Nat Commun, 2018, 9: 717

10 Doyle DA, Morais Cabral J, Pfuetzner RA, et al. The structure of the potassium channel: molecular basis of $\mathrm{K}^{+}$conduction and selectivity. Science, 1998, 280: 69-77

11 MacKinnon R. Potassium channels and the atomic basis of selective ion conduction (Nobel Lecture). Angew Chem Int Ed, 2004, 43: 4265-4277

12 Tadross MR, Dick IE, Yue DT. Mechanism of local and global Ca ${ }^{2+}$ sensing by calmodulin in complex with a $\mathrm{Ca}^{2+}$ channel. Cell, 2008, 133: $1228-1240$

13 Xia F, Guo W, Mao Y, et al. Gating of single synthetic nanopores by proton-driven DNA molecular motors. J Am Chem Soc, 2008, 130: $8345-8350$

14 Xiao K, Xie G, Zhang Z, et al. Enhanced stability and controllability of an ionic diode based on funnel-shaped nanochannels with an extended critical region. Adv Mater, 2016, 28: 3345-3350

15 Holt JK, Gyu Park H, Wang Y, et al. Fast mass transport through sub-2-nanometer carbon nanotubes. Science, 2006, 312: 1034-1037

16 Secchi E, Marbach S, Niguès A, et al. Massive radius-dependent flow slippage in carbon nanotubes. Nature, 2016, 537: 210-213

$17 \mathrm{Wu} \mathrm{K}$, Chen Z, Li J, et al. Wettability effect on nanoconfined water flow. Proc Natl Acad Sci USA, 2017, 114: 3358-3363

18 Hummer G, Rasaiah JC, Noworyta JP. Water conduction through the hydrophobic channel of a carbon nanotube. Nature, 2001, 414: 188-190

19 Chen Q, Meng L, Li Q, et al. Water transport and purification in nanochannels controlled by asymmetric wettability. Small, 2011, 7: 2225-2231

20 Zhu Z, Tian Y, Chen Y, et al. Superamphiphilic silicon wafer surfaces and applications for uniform polymer film fabrication. Angew Chem Int Ed, 2017, 56: 5720-5724

21 Nair RR, Wu HA, Jayaram PN, et al. Unimpeded permeation of water through helium-leak-tight graphene-based membranes. Science, 2012, 335: 442-444

22 Guo W, Cao L, Xia J, et al. Energy harvesting with single-ionselective nanopores: a concentration-gradient-driven nanofluidic power source. Adv Funct Mater, 2010, 20: 1339-1344

23 Feng J, Graf M, Liu K, et al. Single-layer $\mathrm{MoS}_{2}$ nanopores as nanopower generators. Nature, 2016, 536: 197-200

24 Siria A, Poncharal P, Biance AL, et al. Giant osmotic energy conversion measured in a single transmembrane boron nitride nanotube. Nature, 2013, 494: 455-458

25 Zhang Z, Kong XY, Xiao K, et al. Engineered asymmetric heterogeneous membrane: a concentration-gradient-driven energy harvesting device. J Am Chem Soc, 2015, 137: 14765-14772

26 Zhang Z, Sui X, Li P, et al. Ultrathin and ion-selective Janus membranes for high-performance osmotic energy conversion. J Am Chem Soc, 2017, 139: 8905-8914

27 Gao J, Guo W, Feng D, et al. High-performance ionic diode membrane for salinity gradient power generation. J Am Chem Soc, 2014, 136: 12265-12272

28 Vabulas RM, Hartl FU. Protein synthesis upon acute nutrient restriction relies on proteasome function. Science, 2005, 310: 19601963

29 Kosuri S, Church GM. Large-scale de novo DNA synthesis: technologies and applications. Nat Methods, 2014, 11: 499-507

30 Kaita S, Yamanaka M, Horiuchi AC, et al. Butadiene polymerization catalyzed by lanthanide metallocene-alkylaluminum complexes with cocatalysts: metal-dependent control of 1,4-cis/trans stereoselectivity and molecular weight. Macromolecules, 2006, 39: 1359-1363

31 Pan X, Fan Z, Chen W, et al. Enhanced ethanol production inside carbon-nanotube reactors containing catalytic particles. Nat Mater, 2007, 6: 507-511

32 Kageyama K. Extrusion polymerization: catalyzed synthesis of crystalline linear polyethylene nanofibers within a mesoporous silica. Science, 1999, 285: 2113-2115

Acknowledgements This work was supported by the National Key R\&D Program of China (2017YFA0206900), and the National Natural Science Foundation of China (21625303).

Author contributions Jiang L proposed the concept of quantumconfined superfluid. Wen L, Tian Y and Jiang L designed and performed the experiments of ionic and molecular transportation. Wen L, Zhang X and Jiang $\mathrm{L}$ wrote the paper and contributed to the general discussion.

Conflict of interest The authors declare that they have no conflict of interest. 

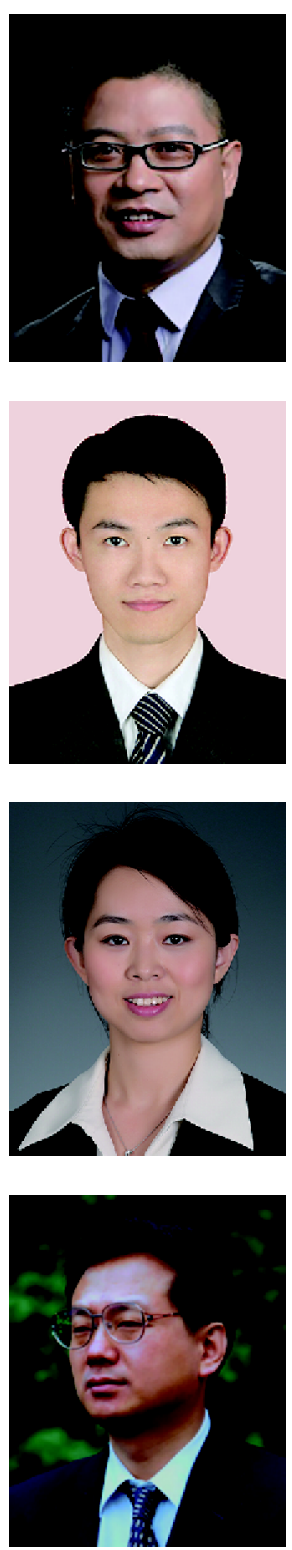

Liping Wen is a Professor at the Technical Institute of Physics and Chemistry, CAS (TIPC). He received his PhD in Physical Chemistry from the Institute of Chemistry, CAS (ICCAS) under the supervision of Prof. Lei Jiang. Since 2010, he has worked as an Associate Professor in ICCAS. In 2011, he went to the Tokyo Institute of Technology as a visiting scholar. In 2015, he moved to the TIPC and was appointed as a Professor in 2016. He was the winner of the National Science Fund for Distinguished Young Scholars in China. Prof. Wen's current scientific interests mainly focus on construction and application of bio-inspired asymmetric smart nanochannel/nanopore membranes in the fields of environment, health, energy, and desalination.

Xiqi Zhang is an assistant professor at the TIPC. He received his BSc (2007), MSc (2009) and PhD (2012) degrees from Sun Yat-sen University under the supervision of Prof. Jiarui Xu and Zhenguo Chi. Afterwards, he worked as a postdoctoral fellow with Prof. Yen Wei at Tsinghua University. In 2014, he joined the TIPC. His current scientific interests are superwettability interfacial materials.

Ye Tian is currently an associate professor at the ICCAS. She received her BSc degree from Northeast Normal University (Changchun, China) in 2006. Then she joined Prof. Lei Jiang's group as a PhD candidate and received her Ph.D. in physical chemistry from ICCAS in 2011. Since 2011, she has worked as an assistant professor (2011.7-2014.2) and an associate professor (2014.2-present) at ICCAS. Her scientific interests focus on superlyophilic interfaces with different dimensions.

Lei Jiang is a Professor at the TIPC and Beihang University. He is an academician of the Chinese Academy of Sciences, Academy of Sciences for the Developing World, and National Academy of Engineering, USA. He received his Bachelor's and Master's degrees from Jilin University, and $\mathrm{PhD}$ from the University of Tokyo. He worked as a post-doctoral fellow with Prof. Akira Fujishima and then as a senior researcher in the Kanagawa Academy of Sciences and Technology with Prof. Kazuhito Hashimoto. In 1999, he joined the ICCAS as part of the Hundred Talents Program. In 2015, he and his group moved to the TIPC. His scientific interests focus on bio-inspired, smart, multi-scale interfacial materials with superwettability.

\section{量子限域超流体: 从自然到人工}

闻利平 ${ }^{1,2}$, 张锡奇 ${ }^{1}$, 田野 ${ }^{2,3}$, 江雷 ${ }^{1,2,4^{*}}$

摘要 生物孔道离子和分子以单链的量子方式快速传输, 我们将其定义为“量子限域超流体”. 限域孔道内离子和分子的有序超流被视为 “量子隧穿流体效应”, 该”隧穿距离”与量子限域超流体的周期相一致. 近期研究表明仿生体系也存在量子限域超流现象, 例如离子通道和 水通道内物质的快速传输. 通过把量子限域超流体概念引入化学领域, 将引发出精准化学合成, 即量子有机、无机、高分子反应等. 而引 入到生物学领域, 将产生量子超流的生物化学、生物物理、生物信息学以及生物医学等. 在此基础上, 也将产生其他的新科学和新技术. 